\title{
Molecular characterization of adenovirus causing acute respiratory disease in Malaysia from 2003 to 2011
}

\author{
Mohd Apandi Y.*, Zarina M. Z., Khairul Azuan O., Nur Ismawati A. R. and Zainah S. \\ Virology Unit, Infectious Disease Research Centre, Institute for Medical Research, Jalan Pahang, 50588 Kuala Lumpur, \\ Malaysia.
}

Accepted 13 August, 2013

\begin{abstract}
Human Adenoviruses (HAdVs) are human pathogens that normally are associated with acute respiratory disease (ARD). Here, in this study, we characterized human adenovirus isolated from respiratory specimens collected from 2003 to 2011. Positive adenovirus were confirmed by cell culture and indirect immunoflourescence (IF) techniques. The isolates and positive clinical samples were subjected to adenovirus polymerase chain reaction, followed by DNA sequencing. BLAST searches and phylogenetic analysis revealed that only 2 species of HAdV, species C at $64 \%(47 / 73)$ and species B at $36 \%(26 / 73)$ were found circulating amongst ARD cases in Malaysia. Four types of HAdV species $C$ were detected namely: HAdV-2 (66\%), HAdV-1(17\%), HAdV-5(13\%) and HAdV-6(4\%). For HAdV species B, only 2 types were detected with HAdV-3 being the highest at $58 \%$ and HAdV-7 at $42 \%$. HadV-7 was associated with severe infections.
\end{abstract}

Key words: Human adenoviruses, acute respiratory disease, molecular characterization, gene sequences.

\section{INTRODUCTION}

Human Adenoviruses (HAdVs) were first isolated from human adenoids and identified as respiratory pathogens in 1953 (Rowe et al., 1953; Hilleman et al., 1954) and now HAdVs have been recognized as aetiological agent for variety of diseases. It is a common infectious agents in children less than 5 years and responsible for 5 to $10 \%$ of all lower respiratory tract infections in infants and children (Choi et al., 2006). They are ubiquitous, non-enveloped, double stranded DNA in the family of Adenoviridae. Diseases in human such as pharyngitis, pneumonia, gastroenteritis, haemorrhagic cystitis and keratoconjunctivitis have been associated with HAdVs infections (Mandell et al., 2009). The severity of the disease also ranges from mild or inapparent clinical syndromes to severe life threatening disease in immunocompromised individuals (Mandell et al., 2009). Infections with HAdVs can occur sporadically or in epidemics; and the virus can be isolated and identified throughout the year (Hong et al., 2001). HAdVs are also responsible for outbreaks in settings that have close living condition such as in military barracks (Dudding et al., 1972), hospital wards (Straube et al., 1983), chronic care facilities (Finn et al., 1988) and police academy (Apandi et al., 2012). Previously, HAdVs were classified by haemagglutination and serum neutralization (Seto et al., 2011). Since genomic data and bioinformatics became available, new HAdVs have been identified including several emerging and recombinant viruses (Walsh et al., 2011) and today there are 57 recognised serotypes in 7 species, HAdV-A to HAdV-G (Martin et al., 2007). The hexon protein with serotype specific encoded by seven hyper variable regions (Crawford and Schnurr, 1996) is the most important components for serotype identification of adenoviruses (Takeuchi et al., 1999).

Epidemiologic characteristics of the HAdV vary by type. All are transmitted by direct contact, faecal oral transmission 
and occasionally waterborne transmission. Depending on the species, these viruses may infect respiratory, conjunctival, gastrointestinal and genitourinary sites and specific types have been linked to distinct clinical syndromes. Recently, HAdV have emerged as life-threatening opportunistic agents in severely immune-suppressed patients, such as stem cell recipients (Ganzenmueller et al., 2011). The outbreak of adenoviruses associated with respiratory disease has been reported worldwide (Schmitz et al., 1983; Zhu et al., 2009; Trei et al., 2010). The serotypes that most frequently associated with acute respiratory disease (ARD) were from species B and C. HAdV-3 and HAdV-7 in species B were reported as a common cause of severe pneumonia in neonates and young children (Carballal et al., 2002; Tsolia et al., 2004); whereas, HAdV-11 and HAdV-14 have been reported in febrile respiratory disease outbreaks in all ages (Kajon et al., 2010; Gu et al., 2012). In the species C, serotypes HAdV1, HAdV-2, HAdV-5 and HAdV-6 were endemic in parts of the world (CDC, 2007) and cause febrile respiratory illnesses in children and young adults (Metzgar et al., 2005; Abd-Jamil et al., 2010). HAdV-4 was the only serotype in species C that involved in ARD (Rubin, 1993; Kandel et al., 2010).

The objectives of the study were to determine the type of HAdV circulating from 2003 to 2011 and the association of HAdV type with severe acute respiratory disease. Therefore, we characterized all HAdVs associated with respiratory illness from samples received by the Institute for Medical Research (IMR) from patients who sought treatment for respiratory infection in the government hospitals in Malaysia.

\section{MATERIALS AND METHODS}

\section{Virus}

From 2003 to 2011, IMR received samples from 10,972 patients who seek treatment at 140 government hospitals for respiratory illness such as acute respiratory distress, pneumonia, bronchiolitis and bronchopneumonia. All samples were screened for respiratory viruses including adenovirus by indirect immunoflourescence assay (IFA respiratory panel $1 \mathrm{Kit}$, Millipore, UK) and cell culture technique. In the IFA technique, samples were considered positive for adenovirus when fluorescence was present in the nucleus or cytoplasm of the cells; whereas in tissue culture solely based on the appearance of the cytopathic effect (CPE). Positive samples by cell culture were recultured in Vero cells and harvested when cells showed CPE.

\section{DNA extraction and PCR}

Viral nucleic acid was extracted from the samples by using Roche high pure viral nucleic acid kit (Roche Applied Science, Mannheim, Germany). Briefly, $200 \mu \mathrm{l}$ of sample was added to binding buffer supplemented with poly $(A)$ and proteinase $K$ in a microcentrifuge tube and incubated at $72^{\circ} \mathrm{C}$ for $10 \mathrm{~min}$. Later, binding buffer was added and the mixture was transferred to high pure filter tube for centrifugation. Inhibitor removal buffer was used to remove PCR inhibitors and wash buffer for removal of residual impurities. Finally, elution buffer was added to elute viral nucleic acid in a clean micro- centrifuge tube. Nested PCR was used to amplify the HAdV hexon gene. First PCR primer pair, forward primer HEXB-1 (5'-AAC ATG ACC AAR GAC TGG TT-3') and reverse primer HEXB-2 (5'-GCC GAG AAS GGY GTR CGC AGG TA -3') (IHCM, 2003) in a one tube reaction $(20 \mu \mathrm{l})$ containing $10 \mu \mathrm{l}$ DNA, $1 \mu \mathrm{l}$ of $20 \mu \mathrm{M}$ of each primer, $2.0 \mu \mathrm{l}$ of $25 \mathrm{mM} \mathrm{MgCl}_{2}$ with 10X PCR buffer, $0.6 \mu \mathrm{l}$ of $10 \mathrm{mM}$ dNTP, $0.5 \mu \mathrm{l}$ of $5 \mathrm{U} / \mu \mathrm{l}$ Taq DNA polymerase and nuclease free water. Samples were subjected to $35 \mathrm{PCR}$ cycles, denaturation at $95^{\circ} \mathrm{C}$ for $30 \mathrm{~s}$, annealing at $60^{\circ} \mathrm{C}$ for $30 \mathrm{~s}$ and extension at $72^{\circ} \mathrm{C}$ for $30 \mathrm{~s}$; and final extension at $72^{\circ} \mathrm{C}$ for $5 \mathrm{~min}$. For the second $\mathrm{PCR}$, forward primer HEXB-3 (5'-TTC AGA AAC TTC CAG CCY ATG AG-3') and reverse primer HEXB-4 (5'-TCC ATG GGA TCC ACC TCA AAR GTC AT-3') (IHCM, 2003) were used and $5 \mu \mathrm{l}$ of first PCR product was used as a template and PCR was performed using the same conditions as the first PCR.

Expected PCR products (360 bp) were examined by gel electrophoresis and QIAquick gel extraction kits (QIAGEN Inc, Valencia, $\mathrm{CA}$ ) was used to extract the DNA from the gel.

\section{Nucleotide sequencing and phylogenetic analysis}

The partial hexon gene amplicons were sequenced on both strands by using primers HEXB-3 and HEXB-4. Sequencing was performed by using the Big Dye Cycle Sequencing kit version 3.0 and an ABI377 automated DNA sequencer (Applied Biosystems, Foster City, CA, USA). The SeqMan software module in the Lasergene suite of programs (DNASTAR, Madison, WI, USA) was used to format the nucleotide sequences. Alignment of the gene sequences was undertaken by using the MegAlign software module in the Lasergene suite of programs (DNASTAR, Madison, WI, USA). Phylogenetic tree was constructed by using the neighbour-joining method from the Software MEGA4 (Tamura et al., 2007). The duck adenovirus type 1 was used as an outgroup for phylogenetic analysis together with different species of adenovirus obtained from GenBank for the purpose of generating dendograms. All sequences were submitted to GenBank with accession number JX182290JX183062.

\section{RESULTS}

More than $76 \%$ specimens found positive for adenoviruses were derived mainly from nasopharyngeal aspirate (NPA) from children less than 5 years of age diagnosed with ARD (Table 1). All 73 positive adenovirus samples, 30 positive by tissue culture and 43 IFA positive were subjected to adenovirus polymerase chain reaction (PCR), and followed by DNA sequencing. Type of samples received, year of isolation, diagnosis and types of adenovirus isolated are shown in Table 1. All positive samples were sequenced, and basic local alignment search tool (BLAST) sequencing analysis (http://blast.ncbi.nlm.nih.gov/Blast.cgi) showed that the sequences belonged to HAdV-1, HAdV-2, HAdV-3, HAdV-5, HAdV-6 and HAdV-7. Phylogenetic tree, constructed on the basis of partial hexon gene (348 bp) nucleotides of all adenoviruses isolated from 2003 to 2011 together with adenovirus isolates retrieved from GenBank is shown in Figure 1. In 2003, only 2 HAdV-7 were detected from ARD specimens and no adenoviruses were detected in 2004. The following year, in 2005, 2 HAdVs were detected and were identified as HAdV-1 and HAdV-2. HAdV detection rate started to increase in 2006, with $10 \mathrm{HAdV}-2$ and $1 \mathrm{HAdV}-1$. The number increased to 
Table 1. Summary of patients' gender, age, sample type, clinical diagnosis, HAdV typing result and GenBank accession number.

\begin{tabular}{|c|c|c|c|c|c|c|}
\hline ID & Accession No. & Sample type & Sex/age & Clinical diagnosis & Isolation year & Adenovirus type \\
\hline RV0227/03 & JX182990 & TA (i) & $\mathrm{M} / 1.3$ & Respiratory distress & 2003 & $\mathrm{Ad} 7$ \\
\hline RV0259/03 & JX182991 & NPA (i) & $\mathrm{M} / 1.5$ & Pneumonia & 2003 & $\mathrm{Ad} 7$ \\
\hline RV0092/05 & JX182992 & NPA (i) & $\mathrm{M} / 2.5$ & Pneumonia & 2005 & Ad1 \\
\hline RV0198/05 & JX182993 & Sputum (i) & $\mathrm{M} / 6$ & Chronic lung Disease & 2005 & Ad2 \\
\hline RV0700/06 & JX182994 & NPA (i) & $\mathrm{M} / 0.3$ & Bronchopneumonia & 2006 & Ad1 \\
\hline RV1007/06 & JX183003 & NPA (p) & $\mathrm{M} / 0.3$ & Pneumonia & 2006 & Ad2 \\
\hline RV1009/06 & JX182995 & NPA (p) & $\mathrm{M} / 0.3$ & Bronchopneumonia & 2006 & Ad2 \\
\hline RV1016/06 & JX182996 & NPA (p) & $\mathrm{M} / 0.6$ & Bronchopneumonia & 2006 & Ad2 \\
\hline RV1027/06 & JX182997 & NPA (p) & $\mathrm{F} / 1.0$ & Severe pneumonia & 2006 & Ad2 \\
\hline RV1067/06 & JX183002 & NPA (p) & $F / 2.4$ & Bronchopneumonia & 2006 & Ad2 \\
\hline RV1083/06 & JX182998 & NPA (p) & $\mathrm{F} / 0.1$ & Bronchopneumonia & 2006 & Ad2 \\
\hline RV1093/06 & JX183004 & NPA (p) & $\mathrm{M} / 0.3$ & Bronchiolitis & 2006 & Ad2 \\
\hline RV1095/06 & JX182999 & NPA (p) & $\mathrm{F} / 1.1$ & Pneumonia & 2006 & Ad2 \\
\hline RV1111/06 & JX183000 & NPA (p) & $\mathrm{M} / 0.3$ & Pneumonia & 2006 & Ad2 \\
\hline RV1115/06 & JX183001 & NPA (p) & $\mathrm{M} / 0.1$ & Pneumonia & 2006 & Ad2 \\
\hline RV0004/07 & JX183005 & NPA (p) & $\mathrm{M} / 1.0$ & Severe bronchopneumonia & 2007 & Ad3 \\
\hline RV0037/07 & JX183006 & NPA (p) & $\mathrm{M} / 0.1$ & Pneumonia & 2007 & Ad2 \\
\hline RV0062/07 & JX183007 & NPA (p) & $\mathrm{M} / 0.5$ & Pneumonia & 2007 & Ad2 \\
\hline RV0114/07 & JX183014 & TA $(p)$ & $\mathrm{M} / 7.0$ & Bronchopneumonia & 2007 & Ad2 \\
\hline RV0353/07 & JX183015 & NPA (p) & $\mathrm{F} / 1.5$ & Pneumonia & 2007 & Ad5 \\
\hline RV0440/07 & JX183019 & NPA (p) & $\mathrm{M} / 1.0$ & Pneumonia & 2007 & Ad1 \\
\hline RV0447/07 & JX183008 & NPA (p) & $\mathrm{M} / 0.1$ & pneumonia & 2007 & Ad2 \\
\hline RV0533/07 & JX183009 & NPA (p) & $\mathrm{M} / 0.8$ & pneumonia & 2007 & Ad3 \\
\hline RV0603/07 & JX183017 & NPA (p) & $F / 6.4$ & Pneumonia & 2007 & Ad2 \\
\hline RV0619/07 & JX183013 & NPA (p) & M0.7 & Bronchopneumonia & 2007 & Ad1 \\
\hline RV1031/07 & JX183010 & NPA (p) & $\mathrm{M} / 0.8$ & Bronchopneumonia & 2007 & Ad1 \\
\hline $\mathrm{RV} 1312 / 07$ & JX183018 & NPA (p) & $\mathrm{M} / 0.5$ & Bronchopneumonia & 2007 & Ad2 \\
\hline RV1426/07 & JX183011 & NPA (i) & $\mathrm{F} / 1.0$ & pneumonia & 2007 & Ad3 \\
\hline RV1601/07 & JX183020 & Sputum (p) & $M / 30$ & AGE with sepsis & 2007 & Ad2 \\
\hline RV1694/07 & $J X 183012$ & NPA (p) & $F / 2.6$ & Acute pharyngotonsilitis & 2007 & Ad2 \\
\hline RV1816/07 & JX183016 & NPA (p) & $\mathrm{M} / 0.8$ & pneumonia & 2007 & Ad2 \\
\hline RV0018/08 & JX183022 & NPA (i) & M/0.6 & Acute bronchiolitis & 2008 & Ad5 \\
\hline RV1293/08 & JX183024 & $\operatorname{NPA}(p)$ & $\mathrm{M} / 0.5$ & Bronchopneumonia & 2008 & Ad3 \\
\hline RV1907/08 & JX183023 & ETS (i) & $\mathrm{F} / 0.5$ & Nosocomial pneumonia & 2008 & Ad2 \\
\hline RV1987/08 & JX183021 & NPA (p) & $\mathrm{M} / 0.5$ & pneumonia & 2008 & Ad3 \\
\hline RV0087/09* & JX183032 & Lung tissue (i) & $M / 52$ & Pneumonia & 2009 & Ad3 \\
\hline RV0224/09 & JX183025 & NPA (p) & $\mathrm{M} / 1.2$ & Pneumonia & 2009 & Ad7 \\
\hline RV0229/09 & JX183033 & NPA (i) & $\mathrm{M} / 1.0$ & Bronchopneumonia & 2009 & Ad3 \\
\hline RV0272/09 & JX183026 & NPA (i) & $\mathrm{M} / 0.8$ & Pneumonia & 2009 & Ad2 \\
\hline RV0322/09 & JX183027 & NPA (i) & $\mathrm{M} / 0.8$ & Pneumonia & 2009 & Ad2 \\
\hline RV0475/09 & JX183028 & NPA (i) & $\mathrm{F} / 0.4$ & Nosocomial pneumonia & 2009 & Ad5 \\
\hline RV0482/09 & JX183029 & NPA (i) & $\mathrm{F} / 0.5$ & Pneumonia & 2009 & Ad1 \\
\hline RV0577/09 & JX183030 & NPA (i) & $\mathrm{F} / 0.4$ & Pneumonia & 2009 & Ad1 \\
\hline RV0725/09 & JX183031 & NPA (i) & $\mathrm{F} / 0.8$ & Pneumonia & 2009 & Ad2 \\
\hline $\mathrm{RP0297/10}$ & JX183036 & $\operatorname{NPA}(p)$ & $\mathrm{M} / 1$ & Bronchopneumonia & 2010 & Ad6 \\
\hline RP0320/10 & JX183034 & ETT (i) & $F / 1$ & Severe pneumonia & 2010 & Ad5 \\
\hline $\mathrm{RP0393/10}$ & JX183035 & TS (i) & $M / 5$ & DHF with pneumonia & 2010 & Ad3 \\
\hline RP0263/11 & JX183054 & $\operatorname{NPA}(p)$ & $M / 1$ & pneumonia & 2011 & Ad3 \\
\hline RP0301/11* & JX183037 & TA $(p)$ & $F / 22$ & CAP with respiratory failure & 2011 & Ad7 \\
\hline
\end{tabular}


Table 1. Contd.

\begin{tabular}{|c|c|c|c|c|c|c|}
\hline RP0302/11* & JX183038 & TA, lung, spleen ( $p$ ) & $\mathrm{F} / 25$ & CAP with respiratory failure & 2011 & $\mathrm{Ad} 7$ \\
\hline RP0320/11 & JX183058 & Stool (i) & $\mathrm{M} / 20$ & CAP & 2011 & Ad5 \\
\hline RP0360/11 & JX183060 & NPA (p) & $\mathrm{M} / 1$ & Acute bronchiolitis & 2011 & Ad1 \\
\hline RP0404/11 & JX183039 & NPA (p) & $F / 3$ & Bronchopneumonia & 2011 & Ad7 \\
\hline RP0423/11 & JX183061 & NPA (i) & $\mathrm{M} / 1.5$ & pneumonia & 2011 & Ad2 \\
\hline RP0460/11 & JX183059 & $\operatorname{NPA}(p)$ & $\mathrm{F} / 0.8$ & pneumonia & 2011 & $\mathrm{Ad} 7$ \\
\hline RP0485/11 & JX183040 & TS (i) & $\mathrm{M} / 24$ & CAP & 2011 & Ad7 \\
\hline RP0507/11 & JX183052 & NPA (i) & $\mathrm{M} / 0.8$ & pneumonia & 2011 & Ad3 \\
\hline RP0510/11 & JX183055 & $\operatorname{NPA}(p)$ & $\mathrm{M} / 17$ & Severe pneumonia & 2011 & Ad7 \\
\hline RP0536/11 & JX183062 & $\operatorname{NPA}(p)$ & $\mathrm{M} / 0.3$ & pneumonia & 2011 & Ad5 \\
\hline RP0540/11 & JX183056 & $\operatorname{NPA}(p)$ & $\mathrm{F} / 0.7$ & Severe pneumonia & 2011 & Ad7 \\
\hline RP0568/11 & JX183041 & NPA (i) & $\mathrm{M} / 4.3$ & pneumonia & 2011 & Ad2 \\
\hline RP0583/11 & JX183042 & $\operatorname{NPA}(p)$ & $\mathrm{F} / 5$ & pneumonia & 2011 & Ad3 \\
\hline RP0592/11 & JX183043 & NPA (p) & $\mathrm{F} / 0.5$ & Acute bronchiolitis & 2011 & $\mathrm{Ad} 7$ \\
\hline RP0612/11 & JX183044 & $\operatorname{NPA}(p)$ & $M / 1.3$ & pneumonia & 2011 & Ad3 \\
\hline RP0622/11 & JX183045 & NPA (i) & $\mathrm{F} / 1.8$ & Severe pneumonia & 2011 & Ad3 \\
\hline RP0630/11 & JX183046 & NPA (i) & $F / 0.5$ & pneumonia & 2011 & Ad3 \\
\hline RP0689/11 & JX183051 & NPA (i) & $\mathrm{M} / 1$ & bronchopneumonia & 2011 & Ad2 \\
\hline RP0695/11 & JX183047 & ETS $(p)$ & $\mathrm{F} / 0.1$ & Respiratory distress & 2011 & Ad6 \\
\hline RP0745/11 & JX183048 & NPA (p) & $\mathrm{M} / 0.1$ & pneumonia & 2011 & Ad3 \\
\hline RP1081/11 & JX183049 & TS (i) & $\mathrm{M} / 23$ & CAP & 2011 & Ad2 \\
\hline RP1082/11 & JX183050 & TS (i) & $M / 22$ & CAP & 2011 & Ad2 \\
\hline RP1095/11* & JX183053 & TS (i) & $\mathrm{M} / 12$ & Meningoencephalitis & 2011 & Ad2 \\
\hline RP1098/11 & JX183057 & TS (i) & $\mathrm{M} / 25$ & $\mathrm{ILI}$ & 2011 & $\mathrm{Ad} 2$ \\
\hline
\end{tabular}

M, Male; F, female; TA, trachea aspirate; NPA, nasopharyngeal aspirate; ETS, endotracheal secretion; TS, throat swab; (i), tissue culture isolate; (p), primary clinical samples; AGE , acute gastroenteritis; DHF , dengue haemorrhagic fever; CAP , community acquired pneumonia; ILI, influenza like illness; *, denotes fatal case

16 in 2007, and 15 (94\%) of the positive PCR samples came from primary clinical specimens. Of the 16 positive samples, 15 were HAdV species $C$ consisting of 9 HAdV2, 3 HAdV-1 and 1 HAdV-5. HAdV-3 from Species B was identified from the remaining $2 \mathrm{HAdVs}$ positive samples. Four HAdV were isolated in 2008, 2 were from primary clinical specimens and were identified as HAdV-3, and 2 from cell culture were identified as HAdV-5 and HAdV-2.

In 2009, from 9 samples found positive for HAdV, 5 different types were identified namely: HAdV-1, HAdV-2, HAdV-3, HAdV-5 and HAdV-7. In 2010, we detected HAdV-6 in species $C$ for the first time from nasopharynxgeal aspirate; together with HAdV-3 and HAdV-5. Most of the positive samples for this study $(\sim 35 \%)$ were received in 2011 where HAdV-7 was the dominant type followed by HAdV-2, HAdV-3, HAdV-5, HAdV-6 and HAdV-1 (Table 1).

\section{DISCUSSION}

HAdV has been recognised as a cause of ARD, gastrointestinal infection and other simple febrile illness (Horwitz, 2001). Specific HAdV types normally present with specific manifestations and severity (Wold and Horwitz, 2007), although there are variations in different parts of the world (Baum, 2005). Young children and immunocompromised patients are the most vulnerable to severe complication of HAdV infections (Kojaoghlanian et al., 2003; Walls et al., 2003). In this study, partial hexon gene sequences were used to characterize the HAdV isolates. This gene region contains the hypervariable region and is the most important components for serotype identification of adenoviruses (Takeuchi et al., 1999). The finding showed that 2 species of HAdV; $64 \%$ of species $C$ and $36 \%$ of species $B$ were found circulating amongst ARD cases in Malaysia from 2003 to 2011. Overrepresentation of HAdV-C at $64 \%$ from total adenovirus detected in the past 9 years could suggest a high prevalence of the virus in the community. This finding was consistent with the report from previous study in Malaysia by Abd-Jamil et al. (2010). She found that $70 \%$ of the HAdV infection from paediatric patients who sought treatment for respiratory tract infection in UMMC, Malaysia from 1999 to 2005 was species $\mathrm{C}$ with HAdV-1 and HAdV-2 were the commonest. These were similar to the findings reported by Garcia et al. (2009) in South America involving 231 characterized adenoviruses collected from influenza likeillness during 2006 to 2008 showed that 161 (76\%) adenoviruses belong to species C, $45(21 \%)$ to species B 


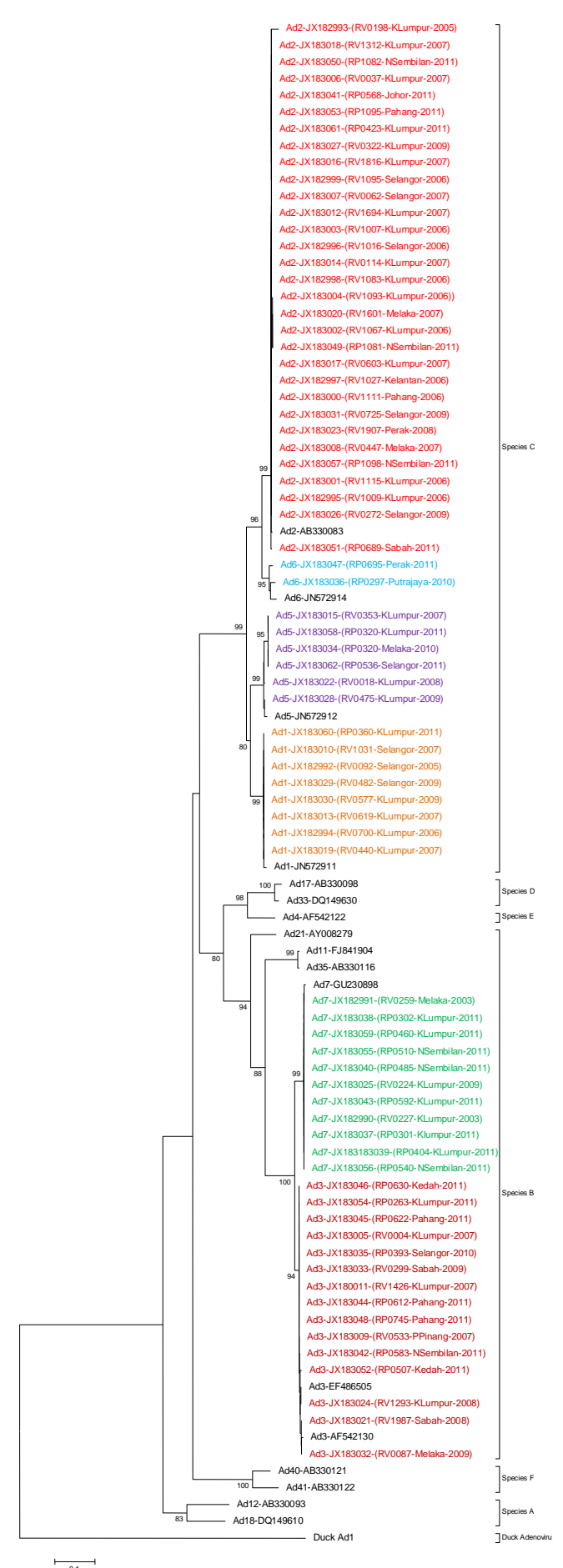

Figure 1. Phylogenetic tree of partial hexon gene sequences (348 bp) of human adenovirus inferred by using the neighbor-joining method from the MEGA4 software (www.megasoftware.net). The evolutionary distances were computed by using the maximum composite likelihood method. Species $A$ to $F$ are indicated by square brackets with duck adenovirus $A$ as an outgroup. Seventy three human adenovirus from acute respiratory disease in Malaysia from 2003 to 2011 are indicated. Representative strains of each species obtained from GenBank are labeled by using the adenovirus species and accession number. Bootstrap values (>75\%) for 1,000 pseudoreplicate datasets are indicated at branch nodes. Scale bar indicates nucleotide substitutions per site. and $7(3 \%)$ to species E. However, a study in Korea reported HAdV species B especially HAdV-3 and HAdV7 , was the predominant serotype among Korean children with respiratory tract infection (Lee et al., 2010).

Similar findings were also reported in Canada (Yeung et al., 2009) and in the military camp in USA (Kajon et al., 2007) where HAdV- B was the major species of HAdV identified from respiratory diseases. The possibility of high prevalence of species C especially HAdV-1, HAdV-2 and HAdV-5, could possibly be due to the virus ability to persist and cause latent infection in tonsils and adenoids of human, which at times cause ARD in young children (Pereira, 1972). Therefore, the prolonged presence of the virus in infected children increases its transmissibility and this could contribute to the persistence presence of HAdVs in young children in Malaysia. HAdV-C species have also been reported not only in respiratory diseases but also in digestive tract infection, regardless of the immune status of the patients and was also found in local ecology in France (Berciaud et al., 2012). In our study, $66 \%$ of HAdV species C detected was HAdV-2 and majority was isolated from children less than 5 years presenting with lower respiratory tract infection such as pneumonia, bronchopneumonia and bronchiolitis (Table 1). Our study showed that only 2 cases of HAdV-2 were related to upper respiratory tract infection (URTI); one isolated from a 25 year-old adult with URTI and another was from a 2.5 year-old child with acute pharyngotonsilitis. Report of fatal outcome associated with HAdV-2 is very rare. However, in this study we found a case of a 12 year-old boy that was admitted for meningoencephalitis, ventilated and died.

No other pathogen such as enterovirus, influenza virus and bacteria were detected except HAdV-2 was isolated from his throat swab. Although, considered as an isolated case, but it could suggest that this type could possibly cause severe infection in patient having underlying disease such as CNS infection. 17\% of the species C detected was HAdV-1 and it was found in children less than 3 years old diagnosed with lower respiratory tract infections (Table 1). This finding is similar to findings from Sevaraju et al. (2011) who reported that HAdV-1 affecting mainly infants and young children less than 2 years of age and caused both upper and lower respiratory infections; and also from Casas et al. (2005) who implicated HAdV-1 with bronchiolitis in children less than 5 years of age. Others adenovirus in this species, HAdV-5 and HAdV-6 were also detected in small numbers in our study and showed to be low prevalence in Malaysia. For HAdV species B, HAdV-3 was more dominant than HAdV-7 at 58 and $42 \%$ detection rate, respectively. Majority of patients with HAdV-3 infection were children less than 5 years old presenting either with pneumonia or severe bronchopneumonia. HAdV-3 was also detected in lung tissue from a fatal case (Table 1), suggesting that type 3 could be associated with severe illnesses and fatal cases. A World Health Organization (WHO) survey on respiratory viral infections reported that HAdV-3 had a 
worldwide distribution and accounted for most of the HAdV associated infections.

Similar situation was also observed in Connecticut (Landry et al., 2009), where an increased incidence of HAdV-3 infections associated with a new variant, HAdV$3 a 51$ that caused mostly mild infections; however, one fatality involving a patient with underlying disease was reported. The same scenario was also observed in Taiwan where HAdV-3 was the commonest HAdV detected among children with RTI from 1999 to 2000 (Hsieh et al., 2009). In our study, HAdV-7 has been found circulating at a low prevalence since 2003 but increased drastically in 2011 with more than $50 \%$ of HAdVs isolated were HAdV-7. The frequency of detection increased as a result of the outbreak of HAdV-7 reported in Malaysia in 2011 where there was an increase in both prevalence and disease severity (Apandi et al., 2012). This was similar to the report from Kansas City, where infections with HAdV-7 increased to 25 from $5.6 \%$ in the previous two years of surveillance and were associated with severe illness such as acute bronchiolitis and pneumonia (Gray et al., 2007). HAdV-7 was also known to cause outbreaks amongst hospitalised children (Choi et al., 2006; Selvaraju et al., 2011), police/army recruit camps (Apandi et al., 2012), and had the potential for morbidity and mortality. HAdV-7 is a well-known pathogen causing epidemics of severe lower respiratory tract infections in children, with a high mortality rate (Baum, 2005). Global survey has shown approximately one-fifth of all HAdV infections reported to World Health Organization (WHO) were attributed to HAdV-7 (Smith et al., 1983; Erdman et al., 2002). All of our HAdV-7 isolates from 2003, 2009 and 2011 belong to HAdV-7d2 which were very similar to the isolate CQ1198 isolated in China in 2010 from children with severe respiratory infection (Ni et al., 2012 unpublished). The same findings were reported by Selvaraju et al. (2011) where HAdV-7 strains 7d2 were responsible for severe lower respiratory tract infection in children in USA and by Tang et al. (2011), where it was associated with infants' pneumonia in China.

In Malaysia, we reported the first HAdV-7 outbreak in 2011 and it was associated with more severe disease and even fatal cases. Most of HAdV-7 isolated in the study was from patients with respiratory distress, severe pneumonia, bronchiolitis and fatal cases due to respiratory failure. These 2 fatal cases were patients related to the outbreak of HAdV-7 in Police Training Centre as reported by Apandi et al. (2012). HAdV-7 has been documented in outbreaks either among infants, older children or adults with serious outcomes (Wadell et al., 1980). The HAdV-7d2 strain was first reported in 1998 by Azar et al. (1998) and since then became the prevalent dominant strain of HAdV-7. In China, HAdV-7d was dominant during 1980 to 1994 and was a representative genome type in Asian until 1998 (Zhang et al., 1986 ) and associated with higher fatality rate than HAdV3 (Li et al., 1996) and Erdman et al. (2002) reported 2 emergent genome types of HAdV-7 and both were associated with epidemic, severe illness and death. Other serotypes from species B, HAdV-11 and HAdV-14 which previously have been associated with acute respiratory disease with fatal outcome (Zhu et al., 2009; Ou et al., 2008), involved in outbreak associated pneumonia (Esposito et al., 2010) and fatal pneumonia (Hong et al., 2001; Tate et al., 2009) were not found in the study.

\section{Conclusion}

This study showed that only 2 species of adenovirus were found circulating among ARD cases in Malaysia in the 9 years period from 2003 to 2011. Majority was HAdV species $C$ at $64 \%$ and the remaining $36 \%(26 / 73)$ was HAdV species B. The predominant type was HAdV type 2, followed by HAdV-3, HAdV-7, HAdV-1, HadV-5 and HAdV-6. HadV-7 was found to be associated with severe clinical presentations.

\section{ACKNOWLEDGEMENTS}

The authors would like to thank the Director General of Health and the Director of the Institute for Medical Research for permission to publish this paper. We would like to acknowledge the staff of the Virology unit in IMR for their technical contributions and laboratory staff from the government hospitals in Malaysia for sending the specimens to IMR. This research project was funded under JPP 11-024.

\section{REFERENCES}

Abd-Jamil J, Teoh BT, Hassan EH, Nuruliza R, Sazaly AB (2010). Molecular identification of adenovirus causing respiratory tract infection in paediatricpatients at the University of Malaya Medical Center. BMC Paed. 10:46.

Apandi Y, Tengku RTAR, Thayan R, Khairul AO, Norhasnida AH, Norfaezah A, Zainah S (2012). Human adenovirus type 7 outbreak in Police Training Center, Malaysia, 2011. Emerg. Infec. Dis: 18(5): 852-854.

Azar R, Varsano M, Mileguir F, Mendelson E (1998). Molecular epidemiology of adenovirus type 7 in Israel: Identification of two new genome types, Ad7k and Ad7d2. J. Med. Virol. 54:291-299.

Baum SG (2005). Adenovirus. In: Mandell GL, Bennett JE, Dolin R, editors. Mandell, Douglas, and Bennett's principles and practice of infectious diseases. 6th ed. Philadelphia, PA: Elsevier Churchill Livingstone. pp. 1835-41.

Berciaud S, Rayne F, Kassab S, Jubert C, Faure-Della CM, Salin F, Wodrich H, Lafon ME (2012). Typadeno Study Members. Adenovirus infections in Bordeaux University Hospital 2008-2010: Clin. virol. features. J. Clin. Viro. 54: 302- 307.

Carballal G, Videla C, Misirlian A, Requeijo PV, Aguilar MC (2002). Adenovirus type 7 associated with severe and fatal acute lower respiratory infections in Argentine children. BMC Pediatr, 2: pg. 6.

Casas I, Avellon A, Mosquera M, Jabado O, Echevarria JE, Campos $\mathrm{RH}$, Rewers M, Perez-Breña P, Lipkin WI, Palacios GI (2005). Molecular identification of adenoviruses in clinical samples by analyzing a partial hexon genomic region. J. Clin. Microbiol. Dec; 43(12):6176-82.

CDC (2007). Acute respiratory disease associated with adenovirus serotype 14-Four states, 2006-200. Morb. Mortal weekly Rep. 
$56: 1181-4$

Choi EH, Lee HJ, Kim SJ, Eun BW, Kim NH, Lee JA, Lee JH, Song EK, Kim SH. (2006). Ten year analysis of adenovirus type 7 molecular epidemiology in Korea, 1994-2004: Implication of fiber diversity. J. Clin. Virol. 35(4):388-393.

Crawford IL, Schnurr DP (1996). Analysis of 15 adenovirus hexon proteins reveals the location and structure of seven hypervariable regions containing serotype-specific residues. J. Virol. 70: 1836-1844.

Dudding BA, Wagner SC, Zellar SC, Gmelith JT, French GR, Top FH (1972). Fatal pneumonia associated with adenovirus type 7 in three military trainees. N Engl. J. Med. 286:1289-1292.

Erdman DD, Xu W, Gerber SI, Gray GC, Schnurr D, Kajon AE, Anderson LJ (2002). Molecular epidemiology of adenovirus type 7 in the United States, 1966-2000. Emerg. Infect. Dis. 8:269-277.

Esposito DH, Gardner TJ, Schneider E, Stockman LJ, Tate JE, Panozzo CA, Robbins CL, Jenkerson SA, Thomas L, Watson CM, Curns AT, Erdman DD, Lu X, Cromeans T, Westcott M, Humphries C, Ballantyne J, Fischer GE, McLaughlin JB, Armstrong G, Anderson LJ (2010). Outbreak of pneumonia associated with emergent human adenovirus serotype 14--Southeast Alaska, 2008. J. Infect. Dis. Jul 15; 202(2):214-22. doi: 10.1086/653498.

Finn A, Andy E, Talbot GH (1988). An epidemic of adenovirus $7 a$ infection in a neonatal nursery:course, morbidity and management. Infect. Control Hosp. Epidemiol. 9:394-404.

Ganzenmueller T, Buchholz S, Harste G, Dammann E, Trenschel R, Heim A (2011). High lethality of human adenovirus disease in adult allogeneic stem cell transplant recipients with high adenoviral blood load. J. Clin. Virol. 2011;52:55-9.

Garcia J, Sovero M, Laguna-Torres VA, Gomez J, Chicaiza W, Barrantes M, Sanchez F, Jimenez M, Comach G, de Rivera IL, Agudo R, Arango AE, Barboza A, Aguayo N, Kochel TJ (2009). Molecular characterization of adenovirus circulating in Central and South America during the 2006-2008 period. Influenza Other Resp. Viruses. 3(6):327-30.

Gray GC, McCarthy T, Lebeck MG, Schnurr DP, Russell KL, Adriana KAE, Landry LM, Leland DS, Storch GA, Ginocchio CC, Robinson CC, Demmler GJ, Saubolle MA, Kehl SC, Selvarangan R, Miller MB, Chappell JD, Zerr DM, Kiska DL, Halstead DC, Capuano AW, Setterquist SF, Chorazy ML, Dawson JD, Erdman DD (2007). Genotype prevalence and risk factors for severe clinical adenovirus infection, United States 2004-2006. Clin. Infect. Dis. 45:1120-31.

Gu L, Liu Z, Li X, Qu J, Guan W, Liu Y, Song S, Yu X, Cao B (2012). Severe community-acquired pneumonia caused by adenovirus type 11 in immunocompetent adults in Beijing. J. Clini. Virol. 54(4): 295301.

Hilleman MR, Werner JH (1954). Recovery of new agent from patients with acute respiratory illness. Proc. Soc. Exp. Biol. Med. 85:183-188.

Hong JY, Lee HJ, Piedra PA, Choi EH, Park KH, Koh YY, Kim WS (2001). Lower respiratory tract infections due to adenovirus in hospitalized Korean children: epidemiology, clinical features, and prognosis. Clin. Infect. Dis. 32: 1423-1429.

Horwitz MS (2001). Adenoviruses. In Fields Virology 4th edition. Edited by: Fields BN, Knipe DM, Howley PM, Griffin DE. Philadelphia: Lippincott Williams \& Wilkins; 2001:2301-2326.

Hsieh WY, Chiu NC, Chi H, Huang FY, Hung CC (2009). Respiratory adenoviral infections in Taiwanese children: a hospital-based study. J. Microbiol. Immunol. Infect. 42(5): 371-377.

Institute of Health and Community Medicine (2003). Standard operating procedure for hand, foot and mouth disease outbreak in UNIMAS Sarawak.

Kajon AE, Dickson LM, Metzgar D, Houng HS, Lee V, Tan BH (2010). Outbreak of febrile repiratory illness associated with adenovirus $11 \mathrm{a}$ infection in a Singapore Military training Camp. J. Clin. Microbiol. 48(4): 438-1441.

Kajon AE, Moseley JM, Metzgar D, Huong HS, Wadleigh A, Ryan MA, Russell KL (2007). Molecular epidemiology of adenovirus type 4 infections in US military recruits in the postvaccination era (19972003). J. Infect. Dis. 196:67-75.

Kandel R, Srinivasan A, D'Agata EM, Lu X, Erdman D, Jhung M (2010). Outbreak of adenovirus type 4 infection in a long term care facility for the elderly. Infect. Cont. Hosp. Epidemiol. 31(7): 755-757.

Kojaoghlanian T, Flomenberg P, Horwitz MS (2003). The impact of adenovirus infection on the immunocompromised host. Rev. Med. Virol. 2003, 13:155-171.

Landry ML, Lebeck MG, Capuano AW, McCarthy T, Gray GC (2009). Adenovirus type 3 outbreak in Connecticut associated with a nove variant. J. Med. Virol. 81(8): 1380-1384.

Lee J, Choi EH, Lee HJ (2010). Comprehensive serotyping and epidemiology of human adenovirus isolated from the repiratory tract of Korean children over 17 consecutive years (1991-2007). J. Med. Virol. 82(4): 624-631.

Li QG, Zheng QJ, Liu YH, Wadell G (1996). Molecular epidemiology of adenovirus type 3 and 7 isolated from children with pneumonia in Beijing. J. Med. Virol. 49:170-177.

Mandell GL, Bennett JE, Dolin R (2009). Mandell, Douglas, and Bennett's principles and practice of infectious diseases, 7th ed Churchill Livingstone/Elsevier, Philadelphia, PA.

Martin MA, Knipe DM, Fields BN, Howley PM, Peter M, Griffin D, Lamb R (2007). Fields' virology. Philadelphia: Wolters Kluwer Health / Lippincott Williams \& Wilkins. Pp. 2395.

Metzgar D, Osuna M, Yingst S, Rakha M, Earhart K, Elyan D, Esmat H, Saad MD, Kajon A, Wu J, Gray GC, Ryan MAK, Russell KL (2005). PCR analysis of Egyptian respiratory adenovirus isolates, including identification of species, serotypes, and coinfections. J. Clin. Microbiol. 43: 5743-5752.

Ou ZY, Zeng QY, Wang FH, Xia HM, Lu JP, Xia JQ, Gong ST, Deng L, Zhang JT, Zhou R (2008). Retrospective study of adenovirus in autopsied pulmonary tissue of pediatric fatal pneumonia in South China. BMC Infect. Dis. 8:122.

Pereira HG (1972). Persistent infection by adenoviruses. J. Clin. Pathol. Suppl. 6:39-42.

Rowe WP, Huebner RJ, Gilmore LK, Parrott RH, Ward TG (1953). Isolation of a cytopathogenic agent from human adenoids undergoing spontaneous degeneration in tissue culture. Proc. Soc. Exp. Biol. Med. 84: 570-573.

Rubin BA (1993). Clinical picture and epidemiology of adenovirus infections. Acta Microbiol Hung 1993, 40:303-323.

Schmitz H, Wigand R, Heinrich W (1983). Worlwide epidemiology of human adenovirus infections. Am. J. Epidemiol. 117: 455-466.

Selvaraju SB, Kovac M, Dickson LM, Kajon AE, Selvarangan R (2011). Molecular epidemiology and clinical presentation of human adenovirus infections in Kansas City Children. J. Clin. Virol. Doi: 10.1016/j.jcv.2011.02/014.

Seto D, Chodosh J, Brister JR, Jones MS (2011). Using the whole genome sequence to characterize and name human adenoviruses. J. Virol. 85:5701-5702.

Straube RC, Thompson MA, Van Dyke RB, Wadell G, Connor JD, Wingaed D, Spector A (1983). Adenovirus type 7b in children's hospital. J. Infect. Dis. 147:814-819.

Takeuchi K, Itoh N, Uchio E, Aoki K, Ohno S (1999). Serotyping of adenoviruses on conjunctival scrapings by PCR and sequence analysis. J. Clin. Microbiol. 37: 1839-1845.

Tamura K, Dudley J, Nei M, Kumar S (2007) MEGA4: Molecular Evolutionary Genetics Analysis (MEGA) software version 4.0. Mol. Biol. Evol. 24:1596-1599.

Tang L, Wang L, Tan X, Xu W (2011). Adenovirus serotype 7 associated with a severe lower respirartory tract disease outbreak in infants in Shaanxi Province, China. Virol. J. 8:23.

Tate JE, Bunning ML, Lott L, Lu X, Su J, Metzgar D, Brosch L, Panozzo CA, Marconi VC, Faix DJ, Prill M, Johnson B, Erdman DD, Fonseca $\mathrm{V}$, Anderson LJ, Widdowson MA (2009). Outbreak of severe respiratory disease associated with emergent human adenovirus serotype 14 at a US air force training facility in 2007. J. Infect. Dis. 199 (10): 1419-26.

Trei JS, Johns NM, Garner JL, Noel LB, Ortman BV, Ensz KL, Johns MC, Bunning ML, Gaydos JC (2010). Spread of adenovirus to geographically dispersed military installations, May-October 2007. EID.www.cdc.gov/eid.16 (5);769-775.

Tsolia MN, Psarras S, Bossios A, Audi H, Paldanius M, Gourgiotis D, Kallergi K, Kafetzis DA, Constantopoulos A, Papadopoulos NG (2004). Etiology of community-acquired pneumonia in hospitalized school-age children: evidence for high prevalence of viral infections. Clin. Infect. Dis. 39(5): 681-686.

Wadell G, Versanyi TM, Lord A, Sutton RN (1980). Epidemic outbreaks 
of adenovirus 7 with special reference to the pathogenicity of adenovirus genome type 7b. Am. J. Epidemiol. 112:619-628.

Walls T, Shankar AG, Shingadia D (2003). Adenovirus: an increasingly important pathogen in paediatric bone marrow transplant patients. Lancet Infect. Dis. 3:79-86.

Walsh MP, Seto J, Liu EB, Dehghan S, Hudson NR, Lukashev AN, Ivanova O, Chodosh J, Dyer DW, Jones MS, Seto D (2011). Computational analysis of two species $C$ human adenoviruses provides evidence of a novel virus. J. Clin. Microbiol. 49(10): 3482-90. doi: 10.1128/JCM.00156-11. Epub 2011 Aug 17.

Wold WS, Horwitz MS (2007). Adenoviruses. In: Knipe DM, Howley PM, editors. Fields virology. 5th ed. Philadelphia, PA: Lippincott Williams \&Wilkins, Inc. 2395-436.
Yeung R, Eshaghi AR, Lombos E, Blair J, Mazzulli T, Burton L, Drews SJ (2009). Characterization of culture-positive adenovirus serotypes from respiratory specimens in Toronto, Ontario, Canada. Virol. J. 6(11): 1-3.

Zhang ZJ, Wang ZL, Cao YP, Zhu ZH, Liu YL, Lin LM, Gao X (1986). Acute respiratory infections in childhood in Beijing: An aetiological study of pneumonia and bronchiolitis. Clin. Med. J. 99:695-702.

Zhu Z, Zhang Y, Xu S, Yu P, Tian X, Wang L, Liu Z, Tang L, Mao N, Ji Y, Li C, Yang Z, Wang S, Wang J, Li D, Xu W (2009). Outbreak of acute respiratory disease in China caused by B2 species of adenovirus type 11. J. Clin. Microbiol. 47(3): 697-703. 